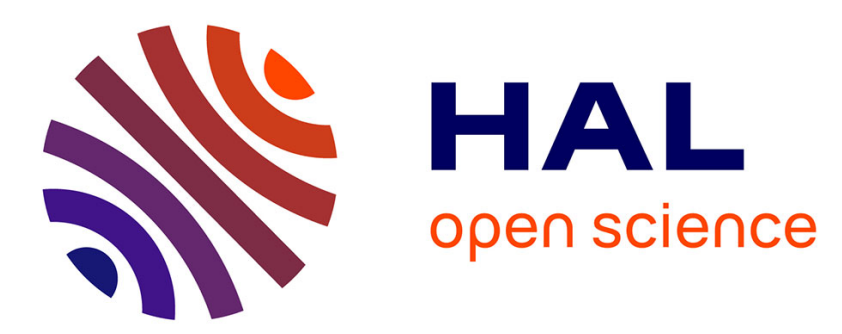

\title{
Characterization of the Singular Part of the Solution of Maxwell's Equations in a Polyhedral Domain
}

Franck Assous, Patrick Ciarlet, Pierre-Arnaud Raviart, Eric Sonnendrücker

\section{To cite this version:}

Franck Assous, Patrick Ciarlet, Pierre-Arnaud Raviart, Eric Sonnendrücker. Characterization of the Singular Part of the Solution of Maxwell's Equations in a Polyhedral Domain. [Research Report] RR-3226, INRIA. 1997, pp.13. inria-00073463

\section{HAL Id: inria-00073463 https://hal.inria.fr/inria-00073463}

Submitted on 24 May 2006

HAL is a multi-disciplinary open access archive for the deposit and dissemination of scientific research documents, whether they are published or not. The documents may come from teaching and research institutions in France or abroad, or from public or private research centers.
L'archive ouverte pluridisciplinaire HAL, est destinée au dépôt et à la diffusion de documents scientifiques de niveau recherche, publiés ou non, émanant des établissements d'enseignement et de recherche français ou étrangers, des laboratoires publics ou privés. 


\title{
Characterization of the singular part of the solution of Maxwell's equations in a polyhedral domain
}

\author{
Franck Assous, Patrick Ciarlet, Pierre-Arnaud Raviart and Eric Sonnendrücker
}

$$
\mathbf{N}^{\circ} 3226
$$

Juillet 1997

THÈME 4 



\title{
Characterization of the singular part of the solution of Maxwell's equations in a polyhedral domain
}

\author{
Franck Assous*, Patrick Ciarlet*, Pierre-Arnaud Raviart ${ }^{\dagger \dagger}$ and Eric Sonnendrücker ${ }^{\ddagger}$ \\ Thème 4 - Simulation et optimisation \\ de systèmes complexes \\ Projet Numath \\ Rapport de recherche $\mathrm{n}^{\circ} 3226$ - Juillet 1997 - 13 pages
}

\begin{abstract}
The solution of Maxwell's equations in a non-convex polyhedral domain is less regular than in a smooth or convex polyhedral domain. In this paper we show that this solution can be decomposed into the orthogonal sum of a singular part and a regular part, and we give a characterization of the singular part. We also notice that the decomposition is linked to the one associated to the scalar Laplacian.
\end{abstract}

Key-words: Maxwell's equations, polyhedral domains, corner singularities.

* CEA Limeil-Valenton, F-94195 Villeneuve-St-Georges

$\dagger$ CMAP, Ecole Polytechnique, F-91128 Palaiseau

$\ddagger$ Institut Elie Cartan, F-54506 Vandœuvre-lès-Nancy

Unité de recherche INRIA Lorraine

Technopôle de Nancy-Brabois, Campus scientifique, 615 rue de Jardin Botanique, BP 101, 54600 VILLERS LÈS NANCY (France)

Téléphone : (33) 0383593030 - Télécopie : (33) 0383278319

Antenne de Metz, technopôle de Metz 2000, 4 rue Marconi, 55070 METZ

Téléphone : (33) 0387203500 - Télécopie : (33) 0387763977 


\section{Caractérisation de la partie singulière de la solution des équations de Maxwell dans un ouvert polyédrique}

Résumé : La solution des équations de Maxwell dans un ouvert polyédrique non convexe est moins régulière que dans le cas d'un domaine régulier ou convexe. Dans ce papier nous montrons que la solution peut être décomposée en la somme orthogonale d'une partie singulière et d'une partie régulière, et nous donnons une caractérisation de la partie singulière. Nous prouvons également que cette décomposition est liée à celle du laplacien scalaire.

Mots-clé : Equations de Maxwell, domaines polyédriques, singularités de coin. 


\section{Introduction}

When solving Maxwell's equations with regular source terms in a non convex polygonal or polyhedral domain (with Lipschitz-continuous boundary) the solutions, instead of being in $H^{1}(\Omega)^{3}$ as in the case of a convex domain, are only in $H(\operatorname{curl}, \Omega) \cap H(\operatorname{div}, \Omega)$. In the same way, when solving a problem involving the scalar Laplace operator with data in $L^{2}(\Omega)$, the solution instead of being in $H^{2}(\Omega)$ as in the regular case (convex polygonal or polyhedral, or with a smooth boundary) is only in $H^{1+s}(\Omega)$, with $0<s<1$. Grisvard [10] showed that a solution of the scalar Laplace operator in the general case could be decomposed into the sum of a regular part and a singular part. This decomposition is based on a decomposition of $L^{2}(\Omega)$ into the sum of the image space of the regular parts and its orthogonal. In the case of a polygonal domain of $\mathbb{R}^{2}$, Grisvard [11] completely characterized these two spaces; starting from this result, we introduced an orthogonal decomposition of the solution of Maxwell's equations and proposed a method for its numerical computation [2], [3]. This method can be generalized to a polyhedral domain with Lipschitz continuous boundary provided an orthogonal decomposition of $L^{2}(\Omega)$ can be obtained and each of its terms can be fully described.

In this article we would like to generalize the results obtained in [2], [3] to the three dimensional case, proving in particular that the solution of Maxwell's equations can also be decomposed into the orthogonal sum of a regular term and a singular term. We shall show that this decomposition is still linked to the decomposition of $L^{2}(\Omega)$ associated to the scalar Laplace operator.

The article is organized as follows. First, we shall recall the characterization of the orthogonal decomposition of $L^{2}(\Omega)$ in the case of a non convex polyhedral domain $\Omega$ with Lipschitz continuous boundary obtained by Assous and Ciarlet [1]. Then for a model problem associated to the steady-state Maxwell equations, we shall introduce a decomposition of the space of solutions, which will enable us to characterize the singular solutions as well as the regular solutions.

In the last part, we obtain results that are complementary to those obtained by Costabel and Dauge [6], who worked on the explicit study of the singular part of the solution. In this spirit, Bonnet-Ben Dhia et al [4] have recently worked on the solution of the frequential Maxwell equations by a regularizing method that can be related to the theory developed by Costabel and Dauge. The originality of our approach lies on the one hand on the theoretical analysis of the decomposition of $L^{2}(\Omega)$ and, on the other hand, in the introduction of orthogonal decompositions, which enable us among other things to describe the space of singular solutions more precisely.

\section{A characterization of the orthogonal of $\Delta\left(H^{2}(\Omega) \cap H_{0}^{1}(\Omega)\right)$ in $L^{2}(\Omega)$}

Let $\Omega$ be a connected and simply connected polyhedral open set of $\mathbb{R}^{3}$ with a connected and Lipschitzcontinuous boundary $\Gamma$. We denote by $\left(\Gamma_{i}\right)_{1 \leq i \leq N_{F}}$ the faces of $\Gamma$.

As we mentionned in the introduction, the space $L^{2}(\Omega)$ can be decomposed in the following way.

Theorem 2.1 The image by the Laplace operator of the space $H^{2}(\Omega) \cap H_{0}^{1}(\Omega)$, denoted by $\Delta\left(H^{2}(\Omega) \cap\right.$ $H_{0}^{1}(\Omega)$ ), is a closed subspace of $L^{2}(\Omega)$, and we have the following orthogonal decomposition:

$$
L^{2}(\Omega)=\Delta\left(H^{2}(\Omega) \cap H_{0}^{1}(\Omega)\right) \stackrel{\perp}{\oplus} N .
$$

Proof: This result was proved by Grisvard [10], and by Dauge [7].

One of the goals of this paper is to characterize the elements $p$ of $N$. To that aim, we denote by $D(\Delta, \Omega)$ the space $\left\{q \in L^{2}(\Omega) ; \Delta q \in L^{2}(\Omega)\right\}$.

By definition, each face $\Gamma_{j}$ is a polygon, hence its boundary $\partial \Gamma_{j}$ is Lipschitz-continuous. For any point $\mathbf{x} \in \Gamma_{j}$, we denote by $\rho_{j}(\mathbf{x})$ the distance of $\mathbf{x}$ to $\partial \Gamma_{j}$. We then have the following definition (cf [11])

$\operatorname{RR}{ }^{\circ} 3226$ 
Definition 2.1 $\widetilde{H}^{1 / 2}\left(\Gamma_{j}\right)$ is the set of functions $f$ of $H^{1 / 2}\left(\Gamma_{j}\right)$ such that $f / \sqrt{\rho_{j}}$ also belongs to $L^{2}\left(\Gamma_{j}\right)$. We denote by $\|f\|_{\sim, 1 / 2, \Gamma_{j}}=\left(\|f\|_{0, \Gamma_{j}}^{2}+\left\|f / \sqrt{\rho_{j}}\right\|_{0, \Gamma_{j}}^{2}\right)^{1 / 2}$ the associated norm.

Finaly, we denote by $\widetilde{H}^{-1 / 2}\left(\Gamma_{j}\right)$ the dual space of $\widetilde{H}^{1 / 2}\left(\Gamma_{j}\right)$.

Now, let us recall the theorem which is proved in Assous and Ciarlet [1].

Theorem $2.2 p \in N$ if and only if

$$
p \in D(\Delta, \Omega), \quad \Delta p=0, \quad p_{\mid \Gamma_{i}}=0 \text { in } \widetilde{H}^{-1 / 2}\left(\Gamma_{i}\right), \text { for } 1 \leq i \leq N_{F} .
$$

The proof is based on several technical results, which we recall as we shall also need them in the present paper, and on the classical theory developped by Gagliardo [8] and Necas [12]. Let $\Gamma_{j}$ be a fixed face.

Proposition 2.1 (i) The normal trace on $\Gamma_{j}$ mapping, $\mathbf{g} \mapsto \mathbf{g} \cdot \mathbf{n}_{\mid \Gamma_{j}}$, is linear and continuous from $\left\{\mathbf{g} \in H^{1}(\Omega)^{3}, \mathbf{g} \times \mathbf{n}_{\mid \Gamma}=0\right\}$ into $\widetilde{H}^{1 / 2}\left(\Gamma_{j}\right)$.

(ii) The trace of the normal derivative on $\Gamma_{j}$ mapping, $u \mapsto(\partial u / \partial n)_{\mid \Gamma_{j}}$, is linear and continuous from $H^{2}(\Omega) \cap H_{0}^{1}(\Omega)$ into $\widetilde{H}^{1 / 2}\left(\Gamma_{j}\right)$.

Next, we define the space

$$
H_{j}(\Omega)=\left\{v \in H^{2}(\Omega) \cap H_{0}^{1}(\Omega), \quad(\partial u / \partial n)_{\mid \Gamma_{k}}=0, \text { for } k \neq j\right\} .
$$

Proposition 2.2 Let $\mu$ be an element of $\widetilde{H}^{1 / 2}\left(\Gamma_{j}\right)$. Then there exists a lifting $u$ belonging to $H_{j}(\Omega)$ such that

$$
\frac{\partial u}{\partial n}_{\Gamma_{j}}=\mu
$$

Proposition 2.3 For a given face $\Gamma_{j}$, there exists a constant $C\left(\Gamma_{j}\right)$ such that

$$
\forall \mu \in \widetilde{H}^{1 / 2}\left(\Gamma_{j}\right), \quad \exists u \in H_{j}(\Omega),{\frac{\partial u}{\partial n} \Gamma_{j}}=\mu \text { and }\|u\|_{2} \leq C\|\mu\|_{\sim, 1 / 2, \Gamma_{j}} .
$$

Proposition 2.4 The space $H^{2}(\Omega)$ is dense in $D(\Delta, \Omega)$ endowed with the graph norm $\|q\|_{D}=\left\{\|q\|_{0}^{2}+\right.$ $\left.\|\Delta q\|_{0}^{2}\right\}^{1 / 2}$.

Proposition 2.5 When $p$ belongs to $D(\Delta, \Omega)$, we have $p_{\mid \Gamma_{i}} \in \widetilde{H}^{-1 / 2}\left(\Gamma_{i}\right)$ for all $1 \leq i \leq N_{F}$. In addition, for each face $\Gamma_{i}$, there exists $C_{i}>0$ such that

$$
\forall p \in D(\Delta, \Omega),\|p\|_{\sim,-1 / 2, \Gamma_{i}} \leq C_{i}\|p\|_{D}
$$

Moreover, the following integration by parts formula holds

$$
\begin{aligned}
& \forall(p, v) \in D(\Delta, \Omega) \times\left(H^{2}(\Omega) \cap H_{0}^{1}(\Omega)\right), \\
& \int_{\Omega} p \Delta v d \mathbf{x}-\int_{\Omega} v \Delta p d \mathbf{x}=\sum_{i}\left\langle p_{\mid \Gamma_{i}},\left(\frac{\partial v}{\partial n}\right)_{\mid \Gamma_{i}}\right\rangle_{\widetilde{H}^{-1 / 2}\left(\Gamma_{i}\right), \widetilde{H}^{1 / 2}\left(\Gamma_{i}\right)} .
\end{aligned}
$$




\section{Application to the Maxwell equations}

\subsection{The model problem}

Consider

$$
X=\{\mathbf{u} \in H(\mathbf{c u r l}, \Omega) \cap H(\operatorname{div}, \Omega), \mathbf{u} \times \mathbf{n}=0 \text { on } \Gamma\}
$$

the Hilbert space endowed with the canonical inner product of $H(\operatorname{curl}, \Omega) \cap H(\operatorname{div}, \Omega)$ and

$$
V=\{\mathbf{u} \in H(\mathbf{c u r l}, \Omega) \cap H(\operatorname{div}, \Omega), \operatorname{div} \mathbf{u}=0, \mathbf{u} \times \mathbf{n}=0 \text { on } \Gamma\}
$$

the Hilbert space endowed with the canonical inner product of $H(\operatorname{curl}, \Omega) \cap H(\operatorname{div}, \Omega)$.

Given a function $g \in L^{2}(\Omega)$, and a function $\mathbf{f} \in L^{2}(\Omega)^{3}$ verifying $\operatorname{div} \mathbf{f}=0$ and $\mathbf{f} \cdot \mathbf{n}=0$ on $\Gamma$, we consider the following problem:

Find $\mathbf{u} \in H(\operatorname{curl}, \Omega) \cap H(\operatorname{div}, \Omega)$ such that:

$$
\begin{aligned}
\operatorname{curl} \mathbf{u} & =\mathbf{f} \text { in } \Omega \\
\operatorname{div} \mathbf{u} & =g \text { in } \Omega \\
\mathbf{u} \times \mathbf{n} & =0 \text { on } \Gamma .
\end{aligned}
$$

Theorem 3.1 Let $\mathbf{f} \in L^{2}(\Omega)^{3}$ with $\operatorname{div} \mathbf{f}=0$ and $\mathbf{f} \cdot \mathbf{n}=0$ on $\Gamma$ and $g \in L^{2}(\Omega)$. Then problem (4)-(6) admits a unique solution $\mathbf{u} \in H(\operatorname{curl}, \Omega) \cap H(\operatorname{div}, \Omega)$.

Proof: The proof can be based on the theory of mixed problems by introducing a Lagrange multiplier for the divergence condition (5) (see Girault-Raviart [9]).

First, the solution of (4)-(6), if it exists, is unique, because from Weber [13], we know that $\|\mathbf{v}\|_{X}=$ $\left\{\|\operatorname{div} \mathbf{v}\|_{0}^{2}+\|\mathbf{c u r l} \mathbf{v}\|_{0}^{2}\right\}^{1 / 2}$ is a norm equivalent to the canonical norm on $X$. Then, it is also solution of the variational problem (if $p=0$ ):

Find $(\mathbf{u}, p) \in X \times L^{2}(\Omega)$ such that

$$
\begin{aligned}
\int_{\Omega} \operatorname{curl} \mathbf{u} \cdot \operatorname{curl} \mathbf{v} d \mathbf{x}+\int_{\Omega} p \operatorname{div} \mathbf{v} d \mathbf{x} & =\int_{\Omega} \mathbf{f} \cdot \operatorname{curl} \mathbf{v} d \mathbf{x} \quad \forall \mathbf{v} \in X, \\
\int_{\Omega} \operatorname{div} \mathbf{u} q d \mathbf{x} & =\int_{\Omega} g q d \mathbf{x} \quad \forall q \in L^{2}(\Omega) .
\end{aligned}
$$

So there remains to prove that the variational problem (7)-(8) has a unique solution. We shall do this with the help of the inf-sup theory (see for example [9]). From Weber [13], it is clear that the bilinear form $(\mathbf{u}, \mathbf{v}) \mapsto \int_{\Omega} \operatorname{curl} \mathbf{u} \cdot \operatorname{curl} \mathbf{v} d \mathbf{x}$ is coercive on the kernel of the second bilinear form (8), that is $V$. Moreover the inf-sup condition is satisfied. Indeed, Let $q \in L^{2}(\Omega)$. Then taking $\mathbf{v}=\nabla \xi$ with $\xi \in H_{0}^{1}(\Omega)$ such that $\Delta \xi=q$ : we have $\mathbf{v} \in X$ and

$$
\int_{\Omega} q \operatorname{div} \mathbf{v} d \mathbf{x}=\int_{\Omega} q \Delta \xi d \mathbf{x}=\int_{\Omega} q^{2} d \mathbf{x} .
$$

It follows that

$$
\inf _{q \in L^{2}(\Omega)} \sup _{\mathbf{v} \in X} \frac{\int_{\Omega} q \operatorname{div} \mathbf{v} d \mathbf{x}}{\|\mathbf{v}\|_{X}\|q\|_{0}} \geq 1 .
$$

Hence the problem (7)-(8) has a unique solution. Finaly, let $\mathbf{v}=\nabla \xi$ with $\xi \in H_{0}^{1}(\Omega)$ such that $\Delta \xi=p$ : we have $\mathbf{v} \in X$. Then ( 7 ) yields

$$
\int_{\Omega} p^{2} d \mathbf{x}=0
$$

which enables us to conclude. Indeed, (7) becomes

$\operatorname{RR~} n^{\circ} 3226$

$$
\int_{\Omega} \operatorname{curl} \mathbf{u} \cdot \operatorname{curl} \mathbf{v} d \mathbf{x}=\int_{\Omega} \mathbf{f} \cdot \operatorname{curl} \mathbf{v} d \mathbf{x} \quad \forall \mathbf{v} \in X
$$


Whence $\operatorname{curl}(\operatorname{curl} \mathbf{u}-\mathbf{f})=0$ and consequently, there exists $\varphi \in H^{1}(\Omega)$ such that $\mathbf{c u r l} \mathbf{u}-\mathbf{f}=\nabla \varphi$. In particular,

$$
\|\nabla \varphi\|_{0}^{2}=\int_{\Omega} \mathbf{c u r l} \mathbf{u} \cdot \nabla \varphi d \mathbf{x}-\int_{\Omega} \mathbf{f} \cdot \nabla \varphi d \mathbf{x}
$$

As $\mathbf{u} \in X$, the first term cancels by integration by parts. The same is true for the second term, thanks to the hypotheses on $\mathbf{f}$. The conclusion follows.

Remark 3.1 The equation (5) can be brought back to the case $g=0$ by letting $\mathbf{v}=\mathbf{u}-\nabla \psi$, $\psi$ being the unique element of $H_{0}^{1}(\Omega)$ verifying $\Delta \psi=g$. The function $\psi$ verifies a Laplace problem (which has been studied by Grisvard [10]) that can be solved with a classical variational formulation. In order to simplify our presentation we shall suppose in the sequel that $g=0$.

\subsection{Decomposition of the space of solutions}

Let us introduce the space of regular solutions

$$
X_{R}=\left\{\mathbf{v} \in H^{1}(\Omega)^{3}, \mathbf{v} \times \mathbf{n}=0 \text { on } \Gamma\right\}, X_{R, j}=\left\{\mathbf{v} \in X_{R}, \mathbf{v} \cdot \mathbf{n}_{\mid \Gamma_{k}}=0 \text { for } k \neq j\right\}
$$

and

$$
V_{R}=\left\{\mathbf{z} \in H^{1}(\Omega)^{3}, \operatorname{div} \mathbf{z}=0, \mathbf{z} \times \mathbf{n}=0 \text { on } \Gamma\right\} .
$$

Proposition 3.1 The spaces $X_{R}$ and $V_{R}$ are closed respectively in $X$ and $V$.

Proof: Costabel showed in [5] that on the space $X_{R}$ we have the equality of the bilinear forms

$$
\int_{\Omega} \nabla \mathbf{v}: \nabla \mathbf{w} d \mathbf{x}=\int_{\Omega} \operatorname{curl} \mathbf{v} \cdot \operatorname{curl} \mathbf{w} d \mathbf{x}+\int_{\Omega} \operatorname{div} \mathbf{v} \operatorname{div} \mathbf{w} d \mathbf{x} .
$$

This equality remains obviously verified on $V_{R}$. The claimed results are a straightforward consequence.

We have the following results, for a given face $\Gamma_{j}$ :

Proposition 3.2 Let $\mu$ belong to $\widetilde{H}^{1 / 2}\left(\Gamma_{j}\right)$. Then there exists a lifting $\mathbf{v} \in X_{R, j}$ such that

$$
\mathbf{v} \cdot \mathbf{n}_{\mid \Gamma_{j}}=\mu .
$$

Proof: A face $\Gamma_{j}$ being fixed, we consider $\mu \in \widetilde{H}^{1 / 2}\left(\Gamma_{j}\right)$. We assume that the face $\Gamma_{j}$ is imbedded in the plane of equation $x_{3}=0$. The reasoning is done in two stages:

(a) Case of a scalar function.

If we denote by $\tilde{\mu}$ the extension of $\mu$ by 0 on $\Gamma$, we have $\tilde{\mu} \in H^{1 / 2}(\Gamma)$. After Necas [12]:

$$
\exists z_{3} \in H^{1}(\Omega), z_{3 \mid \Gamma}=\tilde{\mu} .
$$

(b) Case of a vector function.

If we take $z_{1}=z_{2}=0$ and denote by $\mathbf{z}=\left(z_{1}, z_{2}, z_{3}\right)^{T}$, we have $\mathbf{z} \in H^{1}(\Omega)^{3}$. By construction,

$$
\mathbf{z}_{\mid \Gamma_{k}}=0 \text { for } k \neq j, \mathbf{z} \times \mathbf{n}_{\mid \Gamma_{j}}=0 \text { and } \mathbf{z} \cdot \mathbf{n}_{\mid \Gamma_{j}}=\mu .
$$

In other terms, $\mathbf{z} \in X_{R, j}$ is a lifting of $\mu$. 
Proposition 3.3 For a given face $\Gamma_{j}$, there exists a constant $C\left(\Gamma_{j}\right)$ such that

$$
\forall \mu \in \widetilde{H}^{1 / 2}\left(\Gamma_{j}\right), \quad \exists \mathbf{v} \in X_{R, j}, \quad \mathbf{v} \cdot \mathbf{n}_{\mid \Gamma_{j}}=\mu \text { and }\|\mathbf{v}\|_{1} \leq C\|\mu\|_{\sim, 1 / 2, \Gamma_{j}} .
$$

Proof: We now consider the mapping:

$$
\begin{gathered}
X_{R, j} \longrightarrow \widetilde{H}^{1 / 2}\left(\Gamma_{j}\right) \\
\mathbf{v} \longmapsto \mathbf{v} \cdot \mathbf{n}_{\mid \Gamma_{j}} .
\end{gathered}
$$

Due to propositions 2.1 and 3.2 respectively, it is linear and continuous on the one hand, and onto on the other hand. Moreover its kernel is $\left\{\mathbf{v} \in X_{R, j}, \mathbf{v} \cdot \mathbf{n}_{\mid \Gamma_{j}}=0\right\}=H_{0}^{1}(\Omega)^{3}$.

The mapping $\mathbf{v} \longmapsto \mathbf{v} \cdot \mathbf{n}_{\mid \Gamma_{j}}$ is hence bijective, linear and continuous from $X_{R, j} / H_{0}^{1}(\Omega)^{3}$ into $\widetilde{H}^{1 / 2}\left(\Gamma_{j}\right)$.

The inverse mapping is thus also continuous thanks to the Banach-Steinhaus theorem. The conclusion follows.

Proposition 3.4 We have the following integration by parts formula:

$$
\begin{aligned}
\forall(p, \mathbf{v}) \in & D(\Delta, \Omega) \times X_{R}, \\
& <\nabla p, \mathbf{v}>_{X_{R}^{\prime}, X_{R}}+\int_{\Omega} p \operatorname{div} \mathbf{v} d \mathbf{x}=\sum_{i}\left\langle p_{\mid \Gamma_{i}}, \mathbf{v} \cdot \mathbf{n}_{\mid \Gamma_{i}}\right\rangle_{\widetilde{H}^{-1 / 2}\left(\Gamma_{i}\right), \widetilde{H}^{1 / 2}\left(\Gamma_{i}\right)} .
\end{aligned}
$$

Proof: Let $p \in D(\Delta, \Omega)$. Due to proposition 2.4 we can choose a sequence $\left(p_{k}\right)_{k}$ of elements of $H^{2}(\Omega)$ such that $p_{k} \rightarrow p$ in $D(\Delta, \Omega)$. For $\mathbf{v} \in X_{R}$, we have the relation

$$
\int_{\Omega} \nabla p_{k} \mathbf{v} d \mathbf{x}+\int_{\Omega} p_{k} \operatorname{div} \mathbf{v} d \mathbf{x}=\sum_{i} \int_{\Gamma_{i}} p_{k \mid \Gamma_{i}} \mathbf{v} \cdot \mathbf{n}_{\mid \Gamma_{i}} d \sigma_{i}
$$

As $\mathbf{v} \cdot \mathbf{n}_{\mid \Gamma_{j}} \in \widetilde{H}^{1 / 2}\left(\Gamma_{j}\right)$ thanks to proposition 2.1, and as we know from (2) that the trace mapping is continuous from $D(\Delta, \Omega)$ onto $\tilde{H}^{-1 / 2}\left(\Gamma_{j}\right)$, we get

$$
\left.\int_{\Gamma_{j}} p_{k \mid \Gamma_{j}} \mathbf{v} \cdot \mathbf{n}_{\mid \Gamma_{j}} d \sigma_{j} \rightarrow<p_{\mid \Gamma_{j}}, \mathbf{v} \cdot \mathbf{n}_{\mid \Gamma_{j}}\right\rangle_{\widetilde{H}^{-1 / 2}\left(\Gamma_{j}\right), \widetilde{H}^{1 / 2}\left(\Gamma_{j}\right)} \cdot
$$

On the other hand, as $\int_{\Omega} p_{k} \operatorname{div} \mathbf{v} d \mathbf{x} \rightarrow \int_{\Omega} p \operatorname{div} \mathbf{v} d \mathbf{x}$, the term $\int_{\Omega} \nabla p_{k} \mathbf{v} d \mathbf{x}$ admits a limit when $k \rightarrow+\infty$. Moreover, for $k \neq l$,

$$
\begin{aligned}
\left|\int_{\Omega}\left(\nabla p_{k}-\nabla p_{l}\right) \mathbf{v} d \mathbf{x}\right| & \leq\left\|p_{k}-p_{l}\right\|_{0}\|\operatorname{div} \mathbf{v}\|_{0}+\sum_{i}\left\|p_{k}-p_{l}\right\|_{\sim,-1 / 2, \Gamma_{i}}\|\mathbf{v} \cdot \mathbf{n}\|_{\sim, 1 / 2, \Gamma_{i}} \\
& \leq C\left\|p_{k}-p_{l}\right\|_{D}\|\mathbf{v}\|_{X},
\end{aligned}
$$

due to proposition 2.5 for $\left(p_{k}-p_{l}\right)$ and proposition 2.1 for $\mathbf{v}$. Thus, $\left(\nabla p_{k}\right)_{k}$ is a Cauchy sequence in the dual space of $X_{R}$. Hence it has a limit in this space. On the other hand,

$$
\nabla p_{k} \rightarrow \nabla p \text { in } H^{-1}(\Omega)^{3} \text {. }
$$

As moreover $H_{0}^{1}(\Omega)^{3} \subset X_{R}$, we have $X_{R}^{\prime} \subset H^{-1}(\Omega)^{3}$ and consequently $\left(\nabla p_{k}\right)_{k}$ converges in $X_{R}^{\prime}$ to $\nabla p$. The conclusion follows.

$\operatorname{RR} n^{\circ} 3226$ 
In the case where the boundary $\Gamma$ is smooth or when the domain $\Omega$ is convex we have the equality $V_{R}=V$. But in our case $V_{R}$ is strictly included in $V$. Let us denote by $V_{S}$ the orthogonal of $V_{R}$ in $V$ for the norm $\mathbf{v} \mapsto\|\mathbf{c u r l} \mathbf{v}\|_{0}$ (which is indeed a norm equivalent to the canonical norm after [13]). We then have the decomposition into a direct orthogonal sum

$$
V=V_{R} \stackrel{\perp}{\oplus} V_{S}
$$

We have the following characterization of the space $V_{S}$ :

Theorem 3.2 Let $\mathbf{u}$ be an element of $V$. Then $\mathbf{u}$ belongs to $V_{S}$ if and only if there exists a $p \in N$, unique, such that curl curl $\mathbf{u}=\nabla p$ in $H_{0}(\operatorname{curl}, \Omega)^{\prime}$.

Theorem 3.2 can be proved in the following way, which takes two steps. First we have the theorem Theorem 3.3 Let $\mathbf{u} \in V$. Then $\mathbf{u}$ belongs to $V_{S}$ if and only if there exists a function $p \in L^{2}(\Omega)$, unique, such that

$$
\int_{\Omega} \operatorname{curl} \mathbf{u} \cdot \operatorname{curl} \mathbf{v} d \mathbf{x}+\int_{\Omega} p \operatorname{div} \mathbf{v} d \mathbf{x}=0 \quad \forall \mathbf{v} \in X_{R} .
$$

Proof: Let $\mathbf{u} \in V$, we have $\mathbf{u} \in V_{S}$ if and only if

$$
\int_{\Omega} \operatorname{curl} \mathbf{u} \cdot \operatorname{curl} \mathbf{z} d \mathbf{x}=0 \quad \forall \mathbf{z} \in V_{R} .
$$

Consider then the linear form $l$ defined by

$$
l: \quad \mathbf{v} \mapsto<l, \mathbf{v}>=\int_{\Omega} \operatorname{curl} \mathbf{u} \cdot \operatorname{curl} \mathbf{v} d \mathbf{x}
$$

defined and continuous on $X_{R}$ which cancels on $V_{R}$. In particular, it is a continuous linear form on $H_{0}^{1}(\Omega)^{3}$ which cancels on

$$
V=\left\{\mathbf{v} \in H_{0}^{1}(\Omega)^{3} ; \operatorname{div} \mathbf{v}=0\right\} .
$$

Due to the de Rham theorem, there exists $p \in L^{2}(\Omega)$ (defined for the moment up to a constant) such that

$$
<l, \mathbf{v}>=-\int_{\Omega} p \operatorname{div} \mathbf{v} d \mathbf{x} \quad \forall \mathbf{v} \in H_{0}^{1}(\Omega)^{3}
$$

that is

$$
\int_{\Omega} \operatorname{curl} \mathbf{u} \cdot \operatorname{curl} \mathbf{v} d \mathbf{x}+\int_{\Omega} p \operatorname{div} \mathbf{v} d \mathbf{x}=0 \quad \forall \mathbf{v} \in H_{0}^{1}(\Omega)^{3} .
$$

Now let $\mathbf{v} \in X_{R}$; first we assume that

$$
\int_{\Omega} \operatorname{div} \mathbf{v} d \mathbf{x}=0
$$

After [9], there exists a function $\mathbf{w} \in H_{0}^{1}(\Omega)^{3}$ such that $\operatorname{div} \mathbf{w}=-\operatorname{div} \mathbf{v}$.

Then the function $\mathbf{v}+\mathbf{w}$ of $X_{R}$ verifies $\operatorname{div}(\mathbf{v}+\mathbf{w})=0$, so that $\mathbf{v}+\mathbf{w} \in V_{R}$. This implies

$$
\int_{\Omega} \operatorname{curl} \mathbf{u} \cdot \operatorname{curl}(\mathbf{v}+\mathbf{w}) d \mathbf{x}=0
$$

that is

$$
\int_{\Omega} \operatorname{curl} \mathbf{u} \cdot \operatorname{curl} \mathbf{v} d \mathbf{x}=-\int_{\Omega} \operatorname{curl} \mathbf{u} \cdot \operatorname{curl} \mathbf{w} d \mathbf{x}=\int_{\Omega} p \operatorname{div} \mathbf{w} d \mathbf{x}=-\int_{\Omega} p \operatorname{div} \mathbf{v} d \mathbf{x}
$$

or

$$
\int_{\Omega} \operatorname{curl} \mathbf{u} \cdot \operatorname{curlv} d \mathbf{x}+\int_{\Omega} p \operatorname{div} \mathbf{v} d \mathbf{x}=0
$$


Let us now consider any function $\mathbf{v} \in X_{R}$. We introduce a function $\phi_{0} \in H^{2}(\Omega) \cap H_{0}^{1}(\Omega)$ such that $\int_{\Omega} \Delta \phi_{0} d \mathbf{x}=1$ and set $\mathbf{v}_{0}=\operatorname{grad} \phi_{0}$. Then,

$$
\mathbf{v}_{0} \in X_{R}, \quad \int_{\Omega} \operatorname{div} \mathbf{v}_{0} d \mathbf{x}=1
$$

We then set

$$
\tilde{\mathbf{v}}=\mathbf{v}-\left(\int_{\Omega} \operatorname{div} \mathbf{v} d \mathbf{x}\right) \mathbf{v}_{0}
$$

so that $\tilde{\mathbf{v}} \in X_{R}$ verifies

$$
\int_{\Omega} \operatorname{div} \tilde{\mathbf{v}} d \mathbf{x}=0
$$

Due to the previous considerations, we have

$$
\int_{\Omega} \operatorname{curl} \mathbf{u} \cdot \operatorname{curl} \tilde{\mathbf{v}} d \mathbf{x}+\int_{\Omega} p \operatorname{div} \tilde{\mathbf{v}} d \mathbf{x}=0
$$

But $\operatorname{curl} \tilde{\mathbf{v}}=\operatorname{curl} \mathbf{v}$ and so

$$
\int_{\Omega} p \operatorname{div} \tilde{\mathbf{v}} d \mathbf{x}=\int_{\Omega} p \operatorname{div} \mathbf{v} d \mathbf{x}-\left(\int_{\Omega} p \operatorname{div} \mathbf{v}_{0} d \mathbf{x}\right) \int_{\Omega} \operatorname{div} \mathbf{v} d \mathbf{x}=\int_{\Omega}(p-\lambda(p)) \operatorname{div} \mathbf{v} d \mathbf{x},
$$

with

$$
\lambda(p)=\int_{\Omega} p \operatorname{div} \mathbf{v}_{0} d \mathbf{x} .
$$

(We notice that $p-\lambda(p)$ is determined uniquely). Replacing $p-\lambda(p)$ by $p$, we finally obtain that

$$
\int_{\Omega}(\operatorname{curl} \mathbf{u} \cdot \operatorname{curl} \mathbf{v}+p \operatorname{div} \mathbf{v}) d \mathbf{x}=0 \quad \forall \mathbf{v} \in X_{R} .
$$

Conversely if $\mathbf{u} \in V$ satisfies this last relation, we have straightforwardly

$$
\int_{\Omega} \operatorname{curl} \mathbf{u} \cdot \operatorname{curl} \mathbf{z} d \mathbf{x}=0 \quad \forall \mathbf{z} \in V_{R}
$$

so that $\mathbf{u} \in V_{S}$. Of course, the uniqueness of $p$ comes immediately from the fact that $\mathbf{u} \in V_{S}$ and the first part of the proof.

The second step of the proof consists in a more explicit characterization of $p$.

Lemma 3.1 Let $\mathbf{u}$ be an element of $H_{0}(\operatorname{curl}, \Omega)$. Then $p \in L^{2}(\Omega)$ verifies

$$
\int_{\Omega}(\operatorname{curl} \mathbf{u} \cdot \operatorname{curl} \mathbf{v}+p \operatorname{div} \mathbf{v}) d \mathbf{x}=0 \quad \forall \mathbf{v} \in X_{R}
$$

if and only if

$$
\text { curlcurlu }=\nabla p \text { in } H_{0}(\operatorname{curl}, \Omega)^{\prime}, \quad \text { and } p \in N .
$$

Proof: Taking $\mathbf{v} \in \mathcal{D}(\Omega)^{3}$, we immediately have $\operatorname{curl} \mathbf{c u r l} \mathbf{u}=\nabla p$, in $H_{0}(\operatorname{curl}, \Omega)^{\prime}$, as $\mathbf{c u r l}(\mathbf{c u r l} \mathbf{u}) \in$ $\operatorname{curl} L^{2}(\Omega)^{3} \subset H_{0}(\mathbf{c u r l}, \Omega)^{\prime}$. Choosing $\mathbf{v}=\nabla \phi$, with a function $\phi \in H^{2}(\Omega) \cap H_{0}^{1}(\Omega)$, we find

$$
\int_{\Omega} p \Delta \phi d \mathbf{x}=0
$$

so that $p \in \Delta\left(H^{2}(\Omega) \cap H_{0}^{1}(\Omega)\right)^{\perp}=N$.

Conversely, if $p \in N$ verifies curl curl $\mathbf{u}=\nabla p$ in $H_{0}(\operatorname{curl}, \Omega)^{\prime}$, we have on the one hand

$\operatorname{RR~} n^{\circ} 3226$

$$
<\operatorname{curl} \operatorname{curl} \mathbf{u}, \mathbf{v}>_{H_{0}(\operatorname{curl})^{\prime}, H_{0}(\operatorname{curl})}=\int_{\Omega} \operatorname{curl} \mathbf{u} \cdot \operatorname{curl} \mathbf{v} d \mathbf{x} \quad \forall \mathbf{v} \in X_{R} .
$$


On the other hand (cf (10) proposition 3.4)

$$
\begin{aligned}
<\nabla p, \mathbf{v}>_{X_{R}^{\prime}, X_{R}} & =-\int_{\Omega} p \operatorname{div} \mathbf{v} d \mathbf{x}+\sum_{i}\left\langle p_{\mid \Gamma_{i}}, \mathbf{v} \cdot \mathbf{n}_{\mid \Gamma_{i}}\right\rangle_{\widetilde{H}^{-1 / 2}\left(\Gamma_{i}\right), \widetilde{H}^{1 / 2}\left(\Gamma_{i}\right)} \\
& =-\int_{\Omega} p \operatorname{div} \mathbf{v} d \mathbf{x} \quad \forall \mathbf{v} \in X_{R} .
\end{aligned}
$$

But, as $X_{R}$ is dense in $H_{0}(\operatorname{curl}, \Omega)$, we have

$$
\forall \mathbf{v} \in X_{R} \quad<\nabla p, \mathbf{v}>_{X_{R}^{\prime}, X_{R}}=<\operatorname{curl} \operatorname{curl} \mathbf{u}, \mathbf{v}>_{H_{0}(\operatorname{curl})^{\prime}, H_{0}(\operatorname{curl})} \cdot
$$

The result follows.

Theorem 3.2 is then a straightforward consequence of theorem 3.3 and of lemma 3.1.

Remark 3.2 We also notice that in our case $X_{R}$ is a genuine subspace of $X$. Let us denote by $X_{S}$ the orthogonal of $X_{R}$ in $X$ for the inner product

$$
(\mathbf{v}, \mathbf{w}) \mapsto \int_{\Omega} \operatorname{curl} \mathbf{v} \cdot \operatorname{curl} \mathbf{w} d \mathbf{x}+\int_{\Omega} \operatorname{div} \mathbf{v} \operatorname{div} \mathbf{w} d \mathbf{x}
$$

We then have the following decomposition into a direct orthogonal sum

$$
X=X_{R} \stackrel{\perp}{\oplus} X_{S} .
$$

The solution of (4)-(6) can be written $\mathbf{u}=\mathbf{u}_{R}^{\prime}+\mathbf{u}_{S}^{\prime}$, with $\left(\mathbf{u}_{R}^{\prime}, \mathbf{u}_{S}^{\prime}\right) \in X_{R} \times X_{S}$. In particular, for any $q \in H^{2}(\Omega) \cap H_{0}^{1}(\Omega), \nabla q$ is orthogonal to $\mathbf{u}_{s}^{\prime}$ for the above inner product. This reads

$$
\int_{\Omega} \Delta q \operatorname{div} \mathbf{u}_{s}^{\prime} d \mathbf{x}=0
$$

Hence the definition of $N$ yields that

$$
\operatorname{div} \mathbf{u}_{S}^{\prime} \in N
$$

Thus, if div $\mathbf{u}=0$, it follows that the divergence of the regular part is singular, i. e. $\operatorname{div} \mathbf{u}_{R}^{\prime} \in N$.

\subsection{Saddle-point formulation}

We now come to a saddle-point formulation in $H^{1}(\Omega)^{3}$ of the problem:

Find $\mathbf{u} \in V$ solution of

$$
\operatorname{curl} u=f .
$$

We have already seen, $\mathrm{cf}(7)-(8)$, that $\mathbf{u}$ is a solution of

$$
\begin{aligned}
\int_{\Omega} \operatorname{curl} \mathbf{u} \cdot \operatorname{curl} \mathbf{v} d \mathbf{x} & =\int_{\Omega} \mathbf{f} \cdot \operatorname{curl} \mathbf{v} d \mathbf{x} \quad \forall \mathbf{v} \in X \\
\int_{\Omega} \operatorname{div} \mathbf{u} q d \mathbf{x} & =0 \quad \forall q \in L^{2}(\Omega) .
\end{aligned}
$$

We use the decomposition

$$
\mathbf{u}=\mathbf{u}_{R}+\mathbf{u}_{S} \quad \mathbf{u}_{R} \in V_{R}, \mathbf{u}_{S} \in V_{S} .
$$

To $\mathbf{u}_{S}$ we associate the unique function $p \in N$ such that

$$
\text { curlcurlu } \mathbf{u}_{S}=\nabla p \text { in } H_{0}(\operatorname{curl}, \Omega)^{\prime} .
$$

We can then characterize the pair $\left(\mathbf{u}_{R}, p\right)$ which consists of the regular part $\mathbf{u}_{R}$ and the function $p$ associated to the singular part $\mathbf{u}_{S}$ of the solution $\mathbf{u}$ as the solution of a saddle-point problem in the space $X_{R} \times L^{2}(\Omega)$. 
Theorem 3.4 The pair $\left(\mathbf{u}_{R}, p\right)$ is the unique solution of the problem:

Find $\left(\mathbf{u}_{R}, p\right) \in X_{R} \times L^{2}(\Omega)$ solution of

$$
\begin{aligned}
\int_{\Omega}\left(\operatorname{curl} \mathbf{u}_{R} \cdot \operatorname{curl} \mathbf{v}-p \operatorname{div} \mathbf{v}\right) d \mathbf{x} & =\int_{\Omega} \mathbf{f} \cdot \operatorname{curl} \mathbf{v} d \mathbf{x} \quad \forall \mathbf{v} \in X_{R}, \\
\int_{\Omega} \operatorname{div} \mathbf{u}_{R} q d \mathbf{x} & =0 \quad \forall q \in L^{2}(\Omega) .
\end{aligned}
$$

Proof: Let us verify that $\left(\mathbf{u}_{R}, p\right)$ indeed is a solution of the previous problem. Due to (16), we have

$$
\int_{\Omega}\left(\operatorname{curl} \mathbf{u}_{R} \cdot \operatorname{curl} \mathbf{v}+\operatorname{curl} \mathbf{u}_{S} \cdot \operatorname{curl} \mathbf{v}\right) d \mathbf{x}=\int_{\Omega} \mathbf{f} \cdot \operatorname{curl} \mathbf{v} d \mathbf{x} \quad \forall \mathbf{v} \in X .
$$

Taking $\mathbf{v} \in X_{R}$ and regarding the definition of $p$, we have

$$
\int_{\Omega}\left(\operatorname{curl} \mathbf{u}_{R} \cdot \operatorname{curl} \mathbf{v}-p \operatorname{div} \mathbf{v}\right) d \mathbf{x}=\int_{\Omega} \mathbf{f} \cdot \operatorname{curl} \mathbf{v} d \mathbf{x} \quad \forall \mathbf{v} \in X_{R}
$$

which proves our claim.

It remains to verify the uniqueness of the solution. If $\left(\mathbf{u}_{R}, p\right) \in X_{R} \times L^{2}(\Omega)$ verifies (18)-(19) with $\mathbf{f}=0$, taking $\mathbf{v}=\mathbf{u}_{R} \in V_{R}$, we obtain $\operatorname{curl} \mathbf{u}_{R}=0$ and so, as $\mathbf{u} \mapsto\|\mathbf{c u r l} \mathbf{u}\|_{0}$ is a norm on $V, \mathbf{u}_{R}=0$. Thus

$$
\int_{\Omega} p \operatorname{div} \mathbf{v} d \mathbf{x}=0 \quad \forall \mathbf{v} \in X_{R}
$$

In particular, $\nabla p=0$, hence $p$ is constant. Whence

$$
p \int_{\Omega} \operatorname{div} \mathbf{v} d \mathbf{x}=0 \quad \forall \mathbf{v} \in X_{R}
$$

so $p=0$.

This is enough to prove theorem 3.4. However, let us now also check the existence and uniqueness of a solution to the saddle-point problem (18)-(19) using the inf-sup theory. First, we have already seen that the bilinear form $\int_{\Omega} \operatorname{curl} \mathbf{u}_{R} \cdot \operatorname{curl} \mathbf{v} d \mathbf{x}$ is coercive on $V_{R}$. Thus, all we need to check is the inf-sup condition:

$$
\sup _{\mathbf{v} \in X_{R}} \frac{\int_{\Omega} p \operatorname{div} \mathbf{v} d \mathbf{x}}{\|\mathbf{v}\|_{1}} \geq \beta\|p\|_{0} \quad \forall p \in L^{2}(\Omega) .
$$

This will follow straightforwardly from

Proposition 3.5 The divergence mapping from $X_{R} \rightarrow L^{2}(\Omega)$ is surjective, i.e.

$$
\operatorname{div} X_{R}=L^{2}(\Omega) \text {. }
$$

Proof: We have the inclusions

$$
H_{0}^{1}(\Omega)^{3} \subset X_{R} \text { and } \nabla\left(H^{2}(\Omega) \cap H_{0}^{1}(\Omega)\right) \subset X_{R} .
$$

Hence, as $L_{0}^{2}(\Omega)=\operatorname{div} H_{0}^{1}(\Omega)^{3}($ see $[9])$,

$$
L_{0}^{2}(\Omega) \subset \operatorname{div} X_{R} \text { and } \Delta\left(H^{2}(\Omega) \cap H_{0}^{1}(\Omega)\right) \subset \operatorname{div} X_{R} .
$$

We also have $\operatorname{div} X_{R} \subset L^{2}(\Omega)$, so

$$
L_{0}^{2}(\Omega)+\Delta\left(H^{2}(\Omega) \cap H_{0}^{1}(\Omega)\right) \subset \operatorname{div} X_{R} \subset L^{2}(\Omega) .
$$

$\operatorname{RR~} n^{\circ} 3226$ 
Moreover, obviously,

$$
L^{2}(\Omega)=L_{0}^{2}(\Omega) \oplus \mathbb{R},
$$

the sum being orthogonal in $L^{2}(\Omega)$.

Then, assuming for the moment that there exists $\phi \in H^{2}(\Omega) \cap H_{0}^{1}(\Omega)$ such that $\Delta \phi$ does not belong to $L_{0}^{2}(\Omega)$, there exists a non vanishing real constant $c$ such that $\Delta \phi=f+c$ with $f \in L_{0}^{2}(\Omega)=$ $\operatorname{div} H_{0}^{1}(\Omega)^{3}$. Hence there exists $\mathbf{g} \in H_{0}^{1}(\Omega)^{3}$ such that $f=\operatorname{div} \mathbf{g}$. Then $\mathbf{y}=\nabla \phi-\mathbf{g}$, which belongs to $X_{R}$, is such that $\operatorname{div} \mathbf{y}=c$. Now as $c$ is different from 0 , any constant is the divergence of an element of $X_{R}$ ( $\mathbf{y}$ multiplied by the appropriate scalar).

Now as we already know that the elements of $L_{0}^{2}(\Omega)$ are the divergence of an element of $X_{R}$, the result follows thanks to the decomposition (21).

To end the proof we now need to prove that there exists indeed $\phi \in H^{2}(\Omega) \cap H_{0}^{1}(\Omega)$ such that $\Delta \phi$ does not belong to $L_{0}^{2}(\Omega)$. For this let us assume that $\Delta\left(H^{2}(\Omega) \cap H_{0}^{1}(\Omega)\right) \subset L_{0}^{2}(\Omega)$ and come to a contradiction:

Take $c \in \mathbb{I} \backslash\{0\}$. Then $c \in L^{2}(\Omega)=\Delta\left(H^{2}(\Omega) \cap H_{0}^{1}(\Omega)\right) \oplus N$. Hence

$$
c=\Delta \phi+p, \quad \phi \in H^{2}(\Omega) \cap H_{0}^{1}(\Omega), p \in N,
$$

and $\|c\|_{0}^{2}=\|\Delta \phi\|_{0}^{2}+\|p\|_{0}^{2}$. We have, because of the hypothesis, $\int_{\Omega} \Delta \phi d \mathbf{x}=0$ and, due to the orthogonality of the decomposition, $\int_{\Omega} p \Delta \phi d \mathbf{x}=0$. Then

$$
\|c\|_{0}^{2}=\int_{\Omega} c(\Delta \phi+p) d \mathbf{x}=\int_{\Omega} c p d \mathbf{x}=\int_{\Omega}(\Delta \phi+p) p d \mathbf{x}=\int_{\Omega} p^{2} d \mathbf{x}=\|p\|_{0}^{2} .
$$

Hence $\|\Delta \phi\|_{0}^{2}=0$, which implies that $c \in N$. Then, from Theorem $2.2,\left.c\right|_{\Gamma_{j}}=0$ which implies $c=0$. This contradicts our previous assumption, thus the proposition is proved.

To conclude, we note that the divergence mapping is continuous from $X_{R}$ to $L^{2}(\Omega)$, and that its kernel is $V_{R}$. Thus, due to the Banach-Steinhaus theorem, the inverse mapping is also continuous from $L^{2}(\Omega)$ to $X_{R} / V_{R}$, which yields (20).

Once the pair $\left(\mathbf{u}_{R}, p\right)$ solution of the saddle-point problem (18)-(19) is obtained, there remains to determine the singular part $\mathbf{u}_{S}$ from $p$, that means solving

$$
\operatorname{curlcurl} \mathbf{u}_{S}=\nabla p \text { in } H_{0}(\operatorname{curl}, \Omega)^{\prime}, \text { with } \mathbf{u}_{S} \in V .
$$

Of course, this problem admits a unique solution due to theorem 3.2. 


\section{References}

[1] F. Assous, P. Ciarlet, Jr. , Une caractérisation de l'orthogonal de $\Delta\left(H^{2}(\Omega) \cap H_{0}^{1}(\Omega)\right)$ dans $L^{2}(\Omega)$, accepted for publication in C. R. Acad. Sc. Paris (July 1997).

[2] F. Assous, P. Ciarlet, Jr., E. Sonnendrücker (1996), Résolution des équations de Maxwell dans un domaine avec un coin rentrant, C. R. Acad. Sc. Paris, t. 323, Série I, 203-208.

[3] F. Assous, P. Ciarlet, Jr., E. Sonnendrücker, Resolution of the Maxwell equations in a domain with reentrant corners, accepted for publication in RAIRO Mathematical Modelling and Numerical Analysis (February 1997).

[4] A.-S. Bonnet-Ben Dhia, C. Hazard et S. Lohrengel, A singular field method for the solution of Maxwell's equations in polyhedral domains, submitted to SIAM J. Math. Analysis (April 1997).

[5] M. Costabel (1991), A Coercive Bilinear Form for Maxwell's Equations, J. Math. Anal. and Appl. 157, 2, 527-541.

[6] M. Costabel et M. Dauge (1997), Singularités des équations de Maxwell dans un polyèdre, C. $R$. Acad. Sc. Paris, t. 324, Série I, 1005-1010.

[7] M. Dauge (1988), Elliptic boundary value problems on corner domains, Lecture Notes in Mathematics, Springer Verlag, Berlin.

[8] E. Gagliardo (1957), Caratterizzazioni delle tracce sulla frontiera relative ad alcune classi di funzioni in $n$ variabili, Rend. Sem. Padova 27, 284-305.

[9] V. Girault, P.-A. Raviart (1986), Finite Element Methods for Navier-Stokes Equations, Springer Series in Computational Mathematics, 1341, Springer Verlag, Berlin.

[10] P. Grisvard (1985), Elliptic problems in nonsmooth domains, Monographs and studies in Mathematics, 24, Pitman, London.

[11] P. Grisvard (1992), Singularities in boundary value problems, RMA 22, Masson, Paris.

[12] J. Necas (1967), Les méthodes directes en théorie des équations elliptiques, Masson, Paris.

[13] C. Weber (1980), A local compactness theorem for Maxwell's equations, Math. Meth. in the Appl. Sci. 2, 12-25. 
Unit`e de recherche INRIA Lorraine, Technop^ole de Nancy-Brabois, Campus scientifique, 615 rue du Jardin Botanique, BP 101, 54600 VILLERS LÈS NANCY

Unit'e de recherche INRIA Rennes, Irisa, Campus universitaire de Beaulieu, 35042 RENNES Cedex

Unit'e de recherche INRIA Rh ^one-Alpes, 655, avenue de l'Europe, 38330 MONTBONNOT ST MARTIN

Unit'e de recherche INRIA Rocquencourt, Domaine de Voluceau, Rocquencourt, BP 105, 78153 LE CHESNAY Cedex

Unit'e de recherche INRIA Sophia-Antipolis, 2004 route des Lucioles, BP 93, 06902 SOPHIA-ANTIPOLIS Cedex

\section{Éditeur}

INRIA, Domaine de Voluceau, Rocquencourt, BP 105, 78153 LE CHESNAY Cedex (France)

http://www.inria.fr

ISSN 0249-6399 